\title{
Engraftment of dopamine neuron precursor cells derived from adult mesenchymal stem cells: preliminary in vivo study
}

\begin{abstract}
The use of stem cells has been proposed as an alternative treatment for certain neurodegenerative disorders. It has also been suggested that pre-differentiated stem cells might provide a better therapeutic option than differentiated or undifferentiated stem cells.

The aim of this preliminary study was to determine if immature dopamine neurons cells are capable to engraft into a Parkinson's Disease rat model. Stem cells from rat bone marrow, undifferentiated or pre-differentiated into dopamine precursor cells were implanted into brains of rats Parkinson Disease model. Histological brain analysis and rat rotational behavior were analyzed 1 and 2 weeks after transplantation. Immuno-histochemical analysis of brains showed positive engraftment of transplanted and survived at last for 3 weeks after transplantation. Rotational behavior showed a significantly decreased $(\mathrm{P}<\mathrm{O} .05)$ on rats transplanted with pre-differentiated cells. We present preliminary evidence that dopamine precursor neurons could be an option for Parkinson's Disease cell therapy.
\end{abstract}

Keywords: dopamine precursor cells, neuro-differentiation, parkinson's disease, mesenchymal stem cells
Volume 3 Issue 3 - 2017

\author{
Judith Zavala, Demetrio Arcos, Jorge E \\ Moreno Cuevas, Maria Teresa Gonzalez- \\ Garza \\ Escuela Nacional de Medicina, Tecnologico de Monterrey, \\ Monterrey, NL, Mexico
}

\begin{abstract}
Correspondence: Maria Teresa Gonzalez Garza, Tecnologico de Monterrey, Morones Prieto 3000 Pte, Colonia Doctores, CP 647 I0, Monterrey, NL, Mexico, Tel (52) 8I 88882223, Fax (52) 8I 8888 2148, Email mtgonzalezgarza@itesm.mx
\end{abstract}

Received:September 09, 2017 | Published: November 10, 2017
Abbreviations: PD, parkinson's diseases; iPSCs, induced pluripotential stem cells; ESCs, embryonic stem cells; MSCs, mesenchymal stem cells; BrdU, bromodeoxyuridine; 6-OHDA, 6-hydroxydopamine; MFB, medial forebrain bundle

\section{Introduction}

Cell therapy has been proposed as an alternative for treatment of neurodegenerative disorder. Parkinson's Diseases (PD) are caused by specific and selective loss of nigrostriatal dopaminergic neurons. ${ }^{1}$ The possibility of replacing those cells has been explored through the transplantation of embryonic stem cells (ESCs), fetal stem cells, induced pluripotential stem cells (iPSCs), and mesenchymal stem cells (MSCs) in animal models and clinical trials., ${ }^{1,2}$ The aim of this study was to explore the possibility of transplanting adult MSCs predifferentiated into dopaminergic neurons and look for their capability to brain engraftment and the possible improvement of PD symptoms in a PD rat model.

MSCs were obtained from bone marrow femurs adult male Wistar rats were culture with DMEM medium or Neuro-induction medium (Figure 1). Undifferentiated and dopamine neuron precursor cells were labeled with bromodeoxyuridine (BrdU) (Sigma-Aldrich, St. Louis, MO). For PD model, animals were unilaterally injected with 6-hydroxydopamine (6-OHDA) $(16 \mu \mathrm{g} / 2.5 \mu \mathrm{L}$ dissolved in ascorbatesaline; Sigma-Aldrich) into the right medial forebrain bundle (MFB) by stereo taxis., ${ }^{4,5}$ On this pilot research, three weeks after the 6-OHDA lesion, the rats were divided into two groups. One group $(\mathrm{n}=2)$ was transplanted with $1 \times 10^{5}$ undifferentiated MSCs suspended in $2.5 \mu \mathrm{L}$ PBS. The second group $(\mathrm{n}=3)$ was transplanted with $1 \mathrm{X} 10^{5}$ dopamine neuron precursor cells suspended in $2.5 \mu \mathrm{L}$ PBS. Cells were transplanted to the same area of the previous 6-OHDA injection. The coordinates of the injections were AP-1.8; ML 1.5 DV 7.3mm (Figure 2).
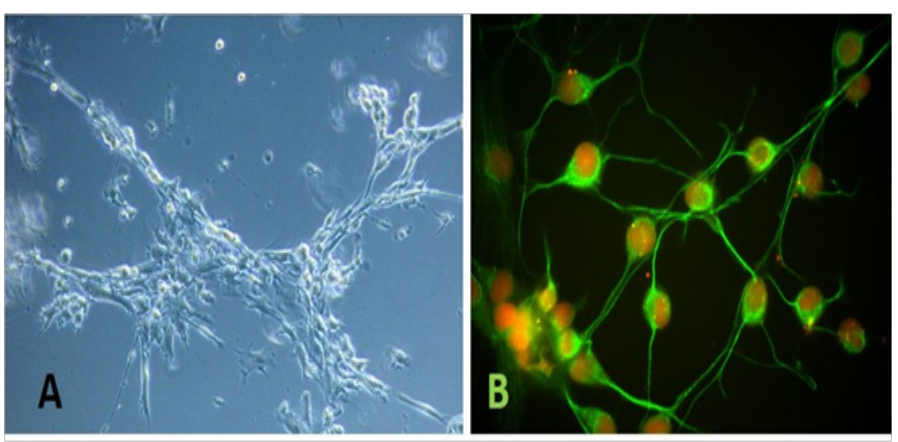

Figure I Microphotograph of mesenchymal stem cells (MSCs) after 24h of cultured in neuro-induction medium. A) Light microphotography of MSCs induced cell showing structural changes (IOX). B) Immunofluorescence microscopy of MSCs cultured in neuro-induction medium. Glial fibrillary acid protein (GFAP) detection (green). Nuclei are counterstained with propidium iodide (red).

\section{Rotational behavior}

To determine any possible beneficial effect of undifferentiated or dopamine neuron precursor cells transplantation, rotational behavior was determined at 1 and 2 weeks after transplantation by intraperitoneal injection of $0.5 \mathrm{mg} / \mathrm{kg}$ apomorphine in $0.9 \%$ ascorbic acid solution ${ }^{6}$ (Sigma-Aldrich). The number of rotations made by each rat was counted for $90 \mathrm{~min}$, beginning 10 min after the injection. Differences in number of rotations between groups were analyzed by the T-student test. 


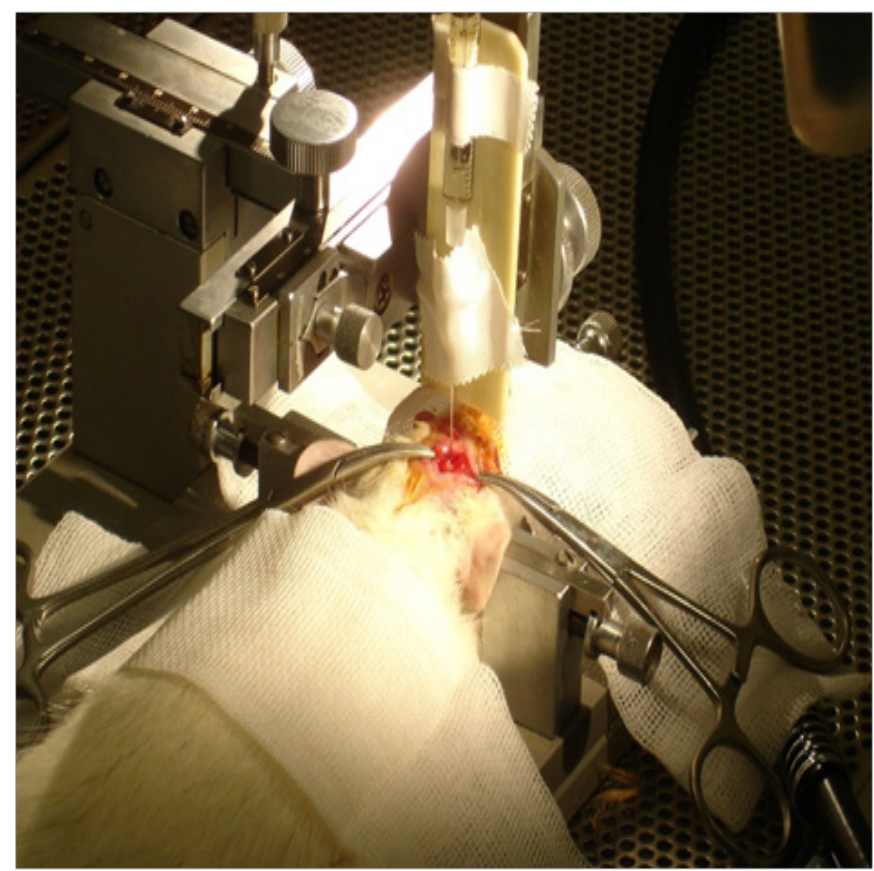

Figure 2 Photography of 6-OHDA injection into medial forebrain bundle (MFB) by stereo taxis4-5 in a Parkinson rat model.

\section{Results}

Immunodetection of MSCs transplanted into rat brains: Three weeks after cell transplantation into the brain, rats were sacrificed and the brains were obtained for immunohistological analysis. ${ }^{7}$ Results confirmed cell engraftment in the brain at the MFB, without migration of the transplanted cells to different areas (Figure 3).

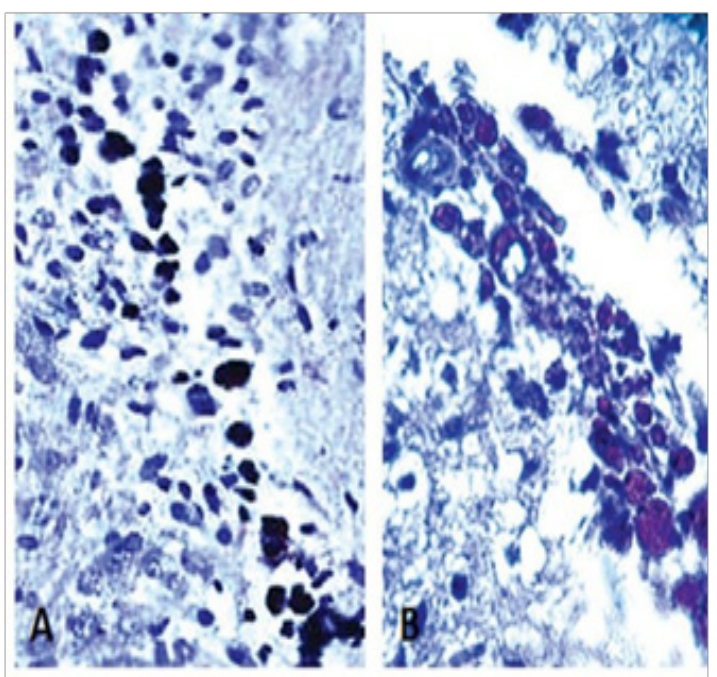

Figure 3 Histological image of a rat brain from the medial forebrain bundle to the $600 \mu \mathrm{m}$ sagittal line. (A) Mesenchymal transplanted cells were detected by an antibody $(\mathrm{Ab})$ to BrdU-peroxidase (brown; I0 X). (B) Histological image of rat brain from the medial forebrain bundle to the $600 \mu \mathrm{m}$ sagittal line $(40 \mathrm{X})$. Transplanted pre-differentiate cells were detected by anti-BrdU Ab.

Apomorphine-induced rotational behavior: Rat rotational behavior was determined 4 and 5weeks after the induction of a Parkinsonian lesion by means of a 6-OHDA injection. After 1 and 2 weeks post- transplantation, animals transplanted with dopamine neuron precursor cells showed a significant decrease in rotational behavior with respect to the baseline $(\mathrm{P}<0.05)$, but no significant decrease in rotational behavior was observed in rats transplanted with undifferentiated MSCs (Figure 4).

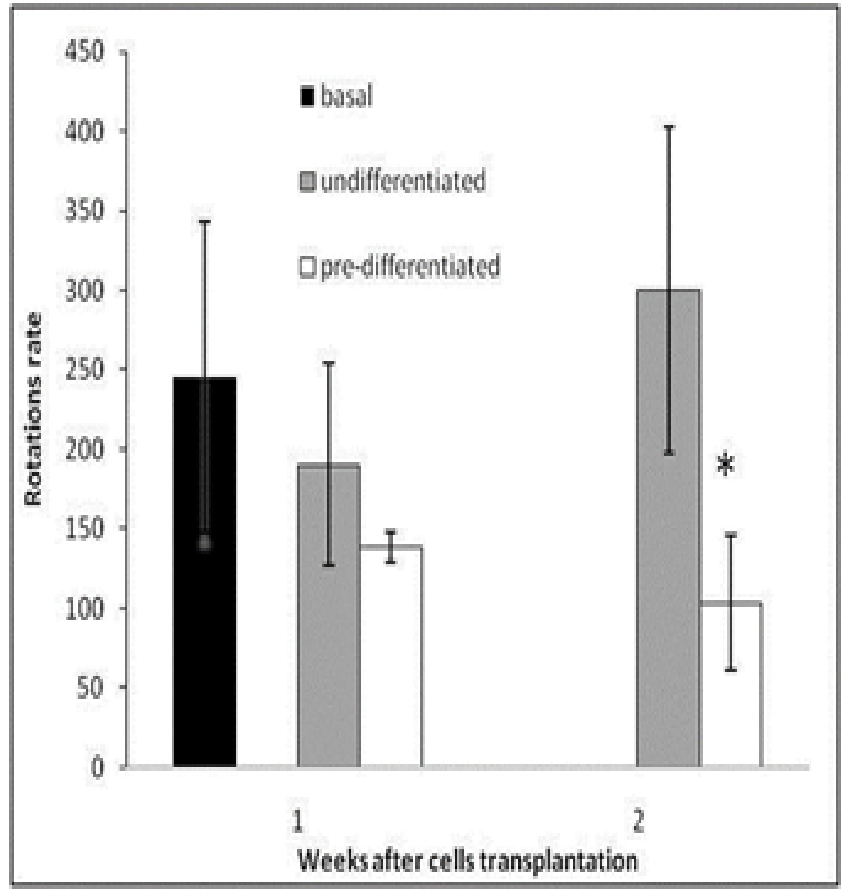

Figure 4 Rotational behavior in rats following apomorphine injection (number of rotations $/ 90 \mathrm{~min}$ ) at I and 2 weeks after MSCs transplantation. (Black column) Rotational rate after 3weeks of 6-hydroxydopamine lesion. (Gray column) Rotational rate of rats transplanted with undifferentiated MSCs. (White column). Rotational rate of rats transplanted with dopamine precursor MSCs $* \mathrm{P}<0.05$.

\section{Discussion}

Transplantation of neural cell aggregates as well as dopaminergic neurons derived from embryonic stem cells in PD animal models have proved to possess potential in improving behavioral tests and cell grafting. ${ }^{7,8}$ However obtaining of human brain neural stem cells for transplantation may limit this procedure as potential therapy. Additionally, the use of embryonic stem cells faces ethical concerns with limitation for their use. Bone marrow mesenchymal stem cell, also has demonstrated to prevent neuronal damage in a PD animal model, ${ }^{9}$ suggesting its potential use as early stage PD therapy. ${ }^{10}$

In this preliminary research the use of neuronal precursor, demonstrate that these cells are capable of engrafting into brain tissue and increased capability of improving behavioral tests compared with bone marrow stem cells alone. In addition the use of dopamine neuron precursor cells obtained in short time periods $3(24 \mathrm{~h})$ reduces contamination probabilities, and epigenetic changes. In order to obtain more conclusive evidence, it will be necessary increase the number of animals in the experimental groups.

\section{Conclusion}

The feasibility of cell isolation (Autologous bone marrow) and harvest (using a single-step pre-differentiation media), along with this 
preliminary results obtained in the animal model, provides evidence of the probable potential of this procedure as therapy for PD. Further studies conducted with longer follow-up will set the guideline to design clinical studies.

\section{Acknowledgements}

The authors express their appreciation to Janet Gutierrez-Alcala and Rosa Maria de la Rosa for technical assistance.

\section{Funding}

This work was partially funded by endowments from ITESM (cat134) and the Zambrano-Hellion Foundation.

\section{Author disclosure statement}

None of the authors has any commercial relationship with any pharmaceutical or biotechnological company. The authors declare that no competing interests exist.

\section{Conflict of interest}

The author declares no conflict of interest.

\section{References}

1. Behari M, Singhal KK. Cell based therapies in Parkinson's Disease. Ann Neurosci. 2011;18(2):76-83.

2. Lescaudron L, Boyer C, Bonnamain V, et al. Assessing the potential clinical utility of transplantations of neural and mesenchymal stem cells for treating neurodegenerative diseases. Methods Mol Biol. 2012;879:147-164.
3. Zavala-Arcos J, Gonzalez-Garza MT, Gutierrez-Alcala J, et al. Direct pre-differentiation of rat mesenchymal stem cells into dopaminergic cells. Stem cell Dis. 2013;3(2):133-138.

4. Smith MP, Cass WA. Oxidative stress and dopamine depletion in an intrastriatal 6-hydroxydopamine model of Parkinson's disease. Neuroscience. 2007;144 (3):1057-1066.

5. Kelsey JE, MagueSD, PijanowskiRS, et al. NMDA receptor antagonists ameliorate the stepping deficits produced by unilateral medial forebrain bundle injections of 6-OHDA in rats. Psychopharmacology. 2004;175(2):179-188.

6. Millan MJ, Maiofiss L, Cussac D, et al. Differential actions of antiparkinson agents at multiple classes of monoaminergic receptor I. A multivariate analysis of the binding profiles of 14 drugs at 21 native and cloned human receptor subtypes. J Pharmacol Exp Ther. 2002;303(2):791-804.

7. Shin ES, Hwang O, Hwang YS, et al. Enhanced efficacy of human brain-derived neural stem cells by transplantation of cell aggregates in a rat model of Parkinson's disease. J Korean Neurosurg Soc. 2014;56(5):383-389.

8. Peng J, Liu Q, Rao MS, et al. Survival and engraftment of dopaminergic neurons manufactured by a Good Manufacturing Practice-compatible process. Cytotherapy. 2014;16(9):1305-1312.

9. Capitelli CS, Lopes CS, Alves AC, et al. Opposite effects of bone marrow-derived cells transplantation in MPTP-rat model of Parkinson's disease: a comparison study of mononuclear and mesenchymal stem cells. Int J Med Sci. 2014;11(10):1049-1064.

10. Suzuki S, Kawamata J, Iwahara N, et al. Intravenous mesenchymal stem cell administration exhibits therapeutic effects against 6-hydroxydopamine-induced dopaminergic neurodegeneration and glial activation in rats. Neurosci Lett. 2015;584:276-281. 